\author{
V.V. Ezhela, V.N. Larin
}

Development of Mathematica Package

'StandardPhysicalConstants'

Contributed paper submitted to the Fifth International Mathematica Symposium (IMS 2003), July 7 - 11, 2003, London, England 


\begin{abstract}
Ezhela V.V., Larin V.N. Development of Mathematica Package 'StandardPhysicalConstants': IHEP Preprint 2003-17. - Protvino, 2003. - p. 6, refs.: 5.

Here we report on the further development of the 'StandardPhysicalConstants' package which was presented for the first time at the last IMS 2001 conference. We would like to dwell on the following issues: the package structure; current status of the physical constant database; data sourses, current data collection and data structure; the main modules of data management system; the first version of "error propagator"; usage examples of one in calculations for high precision tests of physics theories. The outlook of the future development of the package is also given.
\end{abstract}

\title{
Аннотация
}

Ежела В.В., Ларин В.Н. Развитие пакета 'StandardPhysicalConstants' в среде системы Mathematica: Препринт ИФВЭ 2003-17. - Протвино, 2003. - 6 с., библиогр.: 5.

Мы сообщаем о модернизации пакета 'StandardPhysicalConstants', который впервые был представлен на конференции IMS 2001. Описаны структура пакета; текущее состояние базы данных физических постоянных; текущие источники данных, их сбор и структура; основные модули системы управления; первая версия модуля "error propagator"; примеры его использования для высокоточной проверки теоретических параметризаций физических наблюдаемых. Обсуждаются также перспективы дальнейшего развития пакета.

(c) State Research Center of Russia Institute for High Energy Physics, 2003 


\section{The package structure}

The general package structure is not changed much since the first presentation at the previous IMS 2001 [1]. The current version also consists of two files - expanded constant database (Dbfpc110.m) and expanded constant manipulation module (Manager.m). We add to the Manager.m the module ErrorPropagator which is designed to calculate: the average values of algebraic functions depending upon fundamental constants; their variances; standard absolute and relative uncertainties. To simplify manipulation with constants when designing formulae for the physical observables in Mathematica we have created two palettes: the file with package names of the physical constants (CPalette.nb) and the other one with the names of the package functions (FPalette.nb).

\section{Current status of the physical constant database}

The structure of the database is slightly changed as compared to the previous version. We have added one new table with the standard constant names as presented by CODATA [2]. The number of constants stored in the new version of database is increased from 60 to 110 and this is reflected in the name Dbfpc110.m.

\subsection{The data structure}

All data on fundamental physical constants are grouped in the unique list named as dataBase:

$$
\text { dataBase }=\{\text { basicTable, standardNames, ccTable }\},
$$

where: basic Table is the table of the package constant names, constant average values with units, and their standard uncertainties with units; standardNames is the table (list) of the "String" type elements representing the standard CODATA constant names; ccTable is the table of the pair-wise correlation coefficients stored as the list of upper off-diagonal matrix elements of the correlation matrix which is symmetric and real by definition. The structures of the first and third tables are the same as in the first version of the package where they were named as "basicl" and "cct". Only dimensions are increased in each table in accordance with increased number of constants in the database. 


\subsection{Current data collection, sources, and maintenance}

The data set of the current version of the database was collected from the same sources as for the first version: source [2] (unchanged) and source [3] (renewed as of 2002 edition). Unfortunately the data collection still were performed "by hands" for a few reasons. The basic problems are the absence of the data from [3] in a computer readable form, and the "misanthropic manner" of the web access to the correlation data on the CODATA and NIST sites.

Furthermore, in due course of the ErrorPropagator checkout on the real calculations for precision physics we met serious problems with usage the "CODATA recommended values of the fundamental physical constants: 1998" as they presented in both paper and electronic forms.

\subsection{Warning}

It seems that the final CODATA recommended values were seriously damaged on the stage of release and publication preparations and they should be used with great caution. This is a very preliminary warning that we are clarifying. The report on this clarification and our suggestions to improve the situation will be presented elsewhere soon. In the forthcoming sections we will mention the problem once again with an example. Thus, the problem of automated maintenance of the database is postponed to the next iteration of the NIST re-evaluation of the fundamental physical constants.

\section{Current status of the data manipulation package}

Now the data manipulation package consists 17 modules, 6 of which were presented in the first version and described in the previous report [1]. They are also updated, their functional

options remain unchanged but performance are improved. Here we will describe new modules and especially the ErrorPropagator will be presented in detail.

\subsection{Module 'Access'}

This module is the service module that serve for the other modules of the package, but its functions to extract and convert the internal representation of the constants into the standard list might be useful for different applications. Here is an example of usage this module to extract data on muon-electron mass ratio:

$$
\begin{aligned}
& \text { In[3] }:=\text { Access[basicTable, “muonElectronMassRatio"] } \\
& \text { Out }[3]=\left\{206.7682657,6.3 \times 10^{-6}, 3.0 \times 10^{-8}\right\}
\end{aligned}
$$

Here the first element of the list is the average value of the ratio, the second element is the standard uncertainty of the ratio, and the third one is the standard relative uncertainty. Note that in the database these data presented as the string type element with value "206.7682657(63)".

\subsection{Module 'Shift'}

This module is to move specified constant to an another place in the basicTable with simultaneous corresponding rearrangements in the standardNames and ccTable structures. 


\subsection{Module 'PrintDB'}

This module is to printout the whole dataBase or any its part in the conventional form: basicTable, standardNames, ccTable as well as the user defined working sub-base created by the NewListFPC function, being indicated as parameter value of the PrintDB.

\section{Module 'ErrorPropagator'}

The main purpose of the module is to calculate the average value and variance (standard uncertainty) of any recognizable by Mathematica function of any reasonable number of physical constants as variables. In perspective, this module will be able to calculate average values, variances (standard uncertainties), and covariance (correlation) matrix for any recognizable by Mathematica vector-function depending on physical constants.

\subsection{Format}

The ErrorPropagator function has the format:

$$
\text { ErrorPropagator[ formula, database, \{control_keys\}], }
$$

where the first parameter is a formula expressing the dependence of observable (function) upon physical constants, the second parameter is the name of the database used, and the third parameter is the optional list of the control keys. The database can be the main, the working prepared by user on the basis of the main dataBase, or user defined database but with the structure identical to the dataBase.

\subsection{General algorithm}

The general algorithm comprises a few steps:

- Parsing of the formula for observable $\boldsymbol{Q}$, expressed in terms of the physical constant package names from the database to extract the names of constants needed for calculations;

- Forming the temporal working sub-base and extract the corresponding values;

- Calculating the average value $\langle Q\rangle$, variance $\boldsymbol{u}(\boldsymbol{Q})^{2}$, standard uncertainty $\boldsymbol{u}(\boldsymbol{Q})$, and the relative uncertainty $\boldsymbol{u}_{\boldsymbol{r}}(\boldsymbol{Q})=\boldsymbol{u}(\boldsymbol{Q}) /\langle\boldsymbol{Q}\rangle$, if $\langle\boldsymbol{Q}\rangle$ is nonzero.

The results of calculations are collected as the standard list $\left\{\langle Q\rangle, u(Q), u_{r}(Q)\right\}$.

\subsection{Statistical procedure}

Let a physical observable $\boldsymbol{Q}$ depends upon $\boldsymbol{N}$ random variables (physical constants in our case) $\boldsymbol{x}_{\boldsymbol{i}}: \boldsymbol{Q}=\boldsymbol{Q}\left(\boldsymbol{x}_{1}, \boldsymbol{x}_{2}, \ldots, \boldsymbol{x}_{N}\right)$. Then the variance $\boldsymbol{u}(\boldsymbol{Q})^{\mathbf{2}}$ is defined as

$$
u(Q)^{2}=\sum_{i=1}^{N}\left(\frac{\partial Q}{\partial x_{i}}\right)^{2} u\left(c_{i}\right)^{2}+\sum_{i \neq j}^{N} \frac{\partial Q}{\partial x_{i}} \frac{\partial Q}{\partial x_{j}} u\left(c_{i}\right) u\left(c_{j}\right) r_{i j}
$$

where partial derivatives calculated analytically are evaluated at values of $\boldsymbol{x}_{\boldsymbol{i}}=\boldsymbol{c}_{\boldsymbol{i}}, \boldsymbol{u}\left(\boldsymbol{c}_{\boldsymbol{i}}\right)$ is the standard uncertainty of the constant $\boldsymbol{c}_{\boldsymbol{i}}$ and $\boldsymbol{r}_{\boldsymbol{i} \boldsymbol{j}}$ is the corresponding correlation matrix element from the current working database. 
The covariance matrix, if non degenerate, should be positive definite by definition. In case when some of the random quantities are fully correlated $\left(\left|\boldsymbol{r}_{\boldsymbol{i} j}\right|=\mathbf{1}\right)$, the correlation matrix will have zero eigenvalues. Hence, before starting the calculation of the variance the test of the positive definiteness is performed. If matrix is positive definite then the module will produce the standard output. But if the matrix is non positive definite the corresponding warning is generated with printout of the minimal eigenvalue. Calculation is performed in the standard way and then, in addition, all off-diagonal matrix elements are set to zero values and calculation is repeated with identity correlation matrix. In this case the output will be a list with correlation sub-matrix used and two lists of calculated values with and "without" off-diagonal matrix elements.

\subsection{Control parameters}

The third optional parameter in the ErrorPropagator is, in general, a two component list. The first component is a control key to automatic change the values of the temporal correlation matrix elements. Allowed values of the first component are as follows:

- 0 - to set zero value to all off-diagonal matrix elements;

- "Null" - to replace all unknown matrix elements $\boldsymbol{r}_{\boldsymbol{i}}$ (that set to zero value by default) for new "package" variable cC[i,j], for which one can assign any reasonable values;

- $\{\{\boldsymbol{i}, \boldsymbol{j}, \boldsymbol{v a l u e}\}, \ldots\}$ - to replace value of the specified by $(\boldsymbol{i}, \boldsymbol{j})$ correlation coefficient with new value without changing in the temporal correlation matrix.

The second component is to control the output. If it is absent then the output will be the standard one. In the current version the values and meanings of the second component of the control key are as follows:

- " $\boldsymbol{C M}$ " means that in addition to the standard output the correlation matrix used will be presented;

- "EM" means that in addition to the standard output the list of values of the individual terms in the sum defining the variance will be presented to analyze the source of major contributions to the variance;

- "All" means that all above lists will be presented in the output.

\section{Examples of the ErrorPropagator applications}

We produced a few checkout calculations using this module. For the checkout we decided to reproduce some recent calculations [4] of the hyperfine muonium splitting (HFS) and present here only the result for the lowest order (in fine structure constant) term.

\subsection{The muonium hyperfine splitting (HFS)}

The detailed discussion of the re-calculation results will be done elsewhere soon [5]. Here we report that we have reproduced the numerical results obtained in [4] with their values of the relevant fundamental constants without correlations, and failed to reproduce it exactly when using CODATA recommended values with correlations. Just for the illustration we quote the result for the lowest order term calculated by ErrorPropagator

$$
\nu_{F}=\frac{16}{3} \alpha^{2} c R_{\infty} \frac{\mu_{\mu}}{\mu_{B}}\left[\frac{m_{R}}{m_{e}}\right]^{3},
$$


where $\boldsymbol{\alpha}$ is the fine structure constant, $\boldsymbol{c}$ - velocity of light in vacuum, $\boldsymbol{R}_{\infty}-$ Rydberg constant, $\boldsymbol{\mu}_{\boldsymbol{\mu}} / \boldsymbol{\mu}_{\boldsymbol{B}}$ - muon magnetic moment to Bohr magneton ratio, $\boldsymbol{m}_{\boldsymbol{e}}$ - electron mass, $\boldsymbol{m}_{\boldsymbol{\mu}}-$ muon mass, and $\boldsymbol{m}_{\boldsymbol{R}}=\boldsymbol{m}_{e} \boldsymbol{m}_{\boldsymbol{\mu}} /\left(\boldsymbol{m}_{\boldsymbol{e}}+\boldsymbol{m}_{\boldsymbol{\mu}}\right)$.

$$
\begin{aligned}
& \text { In }[4]:=\text { ErrorPropagator }\left[\boldsymbol{\nu}_{\boldsymbol{F}}, \text { dataBase }\right] / \text { Second } \rightarrow \mathbf{1 0}^{\mathbf{3}} \mathbf{k H z}^{\mathbf{1}} \\
& \text { Out }[4]=\left\{4.4590321 \times 10^{6} \mathrm{kHz}, 0.131638 \mathrm{kHz}, 2.95217 \times 10^{-8}\right\}
\end{aligned}
$$

\subsection{Rydberg constant}

As a rule the physical observable might be expressed in terms of physical constants in different algebraically equivalent forms. For the self-consistent values of the constants with proper estimated correlations the numerical results should be statistically consistent. This means that the results can deviate from each other no more than maximal one standard deviation. We have performed a few calculations to check "the re-parameterization consistency" on the CODATA recommended values of the fundamental physical constants (as of 1998 evaluation). One of the evident choice is the Rydberg constant:

$$
\begin{aligned}
& R_{\infty}=\alpha^{2} \frac{m_{e} c_{0}}{2 h} . \\
& \operatorname{In}[5]:=\text { ErrorPropagator }\left[\boldsymbol{R}_{\infty}, \text { dataBase }\right] / \text { Joule } \rightarrow \text { Kilogram } \text { Meter }^{2} \text { Second }^{-2} \\
& / \text {. Meter } \rightarrow 1 \\
& \text { *** WARNING: correlation matrix is non-positive definite! } \\
& \text { Minimum eigenvalue of this matrix is }-0.000425096 \\
& \text { Out }[5]=\{\{\{1 .,-0.092,0.996\},\{-0.092,1 ., 0.002\},\{0.996,0.002,1 .\}\}, \\
& \left\{\left\{1.09737316 \times 10^{7}, 0.0251319 \boldsymbol{i}, 2.29018 \times 10^{-9} \boldsymbol{i}\right\},\right. \\
& \left.\left.\left\{1.09737316 \times 10^{7}, 1.22497,1.11628 \times 10^{-7}\right\}\right\}\right\}
\end{aligned}
$$

(We have removed units here for brevity.) From the results presented it is seen that when calculating with the CODATA values for correlations we obtain nonsence result - the variance is negative (!). In the same time if we ignore correlations then we obtain the standard uncertainty which is five order of magnitude larger than the value quoted by CODATA.

\section{The future of the package}

The considered examples showed that correlations are very important in the precision calculations. We will continue to develop the package to solve remaining problems. There are three major problems:

- The automatic control of the accuracy and precision of the calculations at all stages, and especially in the presentation of the results when vector-functions are involved in the calculations; 
- Organization of the automatic database refreshment after new releases of the CODATA recommended values. The simplest way is to ask NIST or CODATA to create the compact computer readable file without unjustified rounding of the numerical values and accessible by FTP or by Web browsers to be converted into the Mathematica fundamental constant data base;

- Creation of the database containing units and conversion factors. Organization of an automated handling of units during calculations.

In conclusion, let us summarize the preliminary results obtained so far in our activity with fundamental constants in Mathematica:

- The current standard Mathematica package PhysicalConstants is not enough and should be extended to include handling the standard uncertainties and correlations. Our package is aimed to fill this gap;

- The "CODATA recommended values of the fundamental physical constants: 1998" are presented in bad conditions, namely, some non-degenerate sub-matrices of the CODATA correlation matrix published on the Web are non positive definite, presumably because of the unjustified independent rounding of the numerical values of the correlation coefficients;

\section{Acknowledgements}

We thank to V.B. Anikeev and A.D. Ryabov for the clarifying discussions of some aspects and problems with high precision numerical calculations. This work is supported in part by the RFBR grant RFBR-01-07-90392.

\section{References}

[1] Ezhela V. and Larin V. The physical constants for Mathematica, in Proc. of the fourth International Mathematica Symposium, Tokyo, Japan, June, 2001 (eds. Tazawa Y.) June 2001, Tokyo Denki University Press;

IHEP Preprint 2001-27, Protvino, 2001.

[2] Mohr P. and Taylor B. CODATA recommended values of the fundamental physical constants: 1998, in Rev. Mod. Phys., 72, 351 (April 2000) The American Physical Society;

http://physics.nist.gov/cuu/Constants/

[3] Hagiwara K. et al. Review of particle physics. Particle Data Group, in Phys. Rev., D66 010001-1 (July 2002). The American Physical Society.

[4] Czarnecki A., Eidelman S. and Karshenboim S. Muoniun hyperfine structure and hadronic effects, in Phys. Rev., D65, 053004-1 (January 2002) The American Physical Society.

[5] Ezhela V., Larin V. and Siver A. Mathematica System and Precision Tests: Muonium ground-state hyperfine splitting (to be published). 
Препринт отпечатан с оригинала-макета, подготовленного авторами.

В.В. Ежела, В.Н. Ларин

Развитие пакета 'StandardPhysicalConstants' в среде системы Mathematica.

Оригинал-макет подготовлен с помощью системы $\mathrm{A} \mathrm{TE}_{\mathrm{E}} \mathrm{X}$.

Подписано к печати 09.07.2003. Формат $60 \times 84 / 8$.

Офсетная печать. Печ.л. 0.75. Уч.-изд.л. 0.6. Тираж 160.

Заказ 88. Индекс 3649.

ГНЦ РФ Институт физики высоких энергий

142284, Протвино Московской обл. 
Индекс 3649

П Р Е П Р И Н Т 2003-17,

И Ф В Э,

2003 